\title{
Precise attitude determination strategy for spacecraft based on informa- tion fusion of attitude sensors: Gyros/GPS/Star-sensor
}

\author{
MAO Xinyuan*, DU Xiaojing** and FANG Hui*** \\ School of Aerospace Engineering, Beijing Institute of Technology, Beijing, CHINA.
}

\begin{abstract}
The rigorous requirements of modern spacecraft missions necessitate a precise attitude determination strategy. This paper mainly researches that, based on three space-borne attitude sensors: 3-axis rate gyros, 3-antenna GPS receiver and starsensor. To obtain global attitude estimation after an information fusion process, a feedback-involved Federated Kalman Filter (FKF), consisting of two subsystem Kalman filters (Gyros/GPS and Gyros/Star-sensor), is established. In these filters, the state equation is implemented according to the spacecraft's kinematic attitude model, while the residual error models of GPS and star-sensor observed attitude are utilized, to establish two observation equations, respectively. Taking the sensors' different update rates into account, these two subsystem filters are conducted under a variable step size state prediction method. To improve the fault tolerant capacity of the attitude determination system, this paper designs malfunction warning factors, based on the principle of $\chi^{2}$ residual verification. Mathematical simulation indicates that the information fusion strategy overwhelms the disadvantages of each sensor, acquiring global attitude estimation with precision at a 2-arcsecs level. Although a subsystem encounters malfunction, FKF still reaches precise and stable accuracy. In this process, malfunction warning factors advice malfunctions correctly and effectively
\end{abstract}

Key words: Attitude Determination, Multiple Update Rates, Federated Kalman Filter, Information Fusion

\section{Introduction}

In the field of precise attitude determination for spacecraftin near Earth orbit, it is possible to utilize many attitude sensors: 3-Axis Rate Gyros, Star-Sensor, Sun-Sensor, Magnetometer, Infrared Horizon-Sensor, etc. These sensors offer various kinds of information, and thus lead to different attitude determination strategies. Methods such as the optimized TRIAD method [1], Q-method [2], Unscented Kalman Filter (UKF) [3], as well as the FKF method described in this paper, are prevalent. Basically, they are implemented according to the kinematic model of spacecraft attitude motion, achieving information fusion and global optimal estimation. By taking advantage of multi-sensors' information while avoiding their disadvantages, these methods acquire higher attitude determination accuracy, and higher malfunction tolerance capacity. Thus it is possible to obtain attitude estimation that is better than single sensor observation accuracy. Or in other words, to fulfill the same attitude determination accuracy requirement of a system, it presumably decreases the demand in performance indices of each sensor, remarkably reducing the total budget of the attitude determination system [4]. However, the characteristics of different sensors differ greatly. As for the three attitude sensors utilized in this paper: 3-Axis Rate Gyros have excellent performance in a short period, whereas the residuals of observation attitude will accumulate quickly, and result in unacceptable accuracy in the long term; GPS is lower in price and mass, but it will be jammed easily by complicated space circumstance and high-dynamic relative motion between GPS satellites and the spacecraft; and StarSensor is known to be the best attitude sensor, however, it will be interrupted by sunlight, and reflective light from the earth. What is more, it is extremely expensive, exerting great pressure on the attitude determination system budget [5].
This is an Open Access article distributed under the terms of the Creative Commons Attribution Non-Commercial License (http://creativecommons.org/licenses/by$\mathrm{nc} / 3.0 /$ which permits unrestricted non-commercial use, distribution, and reproduction in any medium, provided the original work is properly cited.

\footnotetext{
(c) * Ph. D. Candidate

** Associate-Professor, Corresponding author: duxiaojing2633@163.com *** Postgraduate Student
} 
Another complex problem is the information fusion strategy of sensors. Generally speaking, the Kalman filter is a classical recursive filter, with good performance in dealing with white noise, and achieving optimal estimation [6]. To cope with the fusion process of useful information, Pearson issued the Federated Kalman Filter method, which introduces a process of erecting several subsystem filters, and centralizing the final information fusion step in a main filter, which eventually outputs the global estimation of a complex system [7]. Besides, these sensors have different update rates, bringing unavoidable interference to the ultimate information fusion process. To utilize all these information effectively, a variable step size state prediction Kalman filter is established. It updates and predicts a sensor's state, when the sensor is in update gaps. When all the sensors' observation data are accessible, their data are then allowed to be involved in a filtering process [8].

This paper is organized as follows: In section 2, two subsystem Kalman filters are established. In section 3, an FKF is established to acquire global estimation, and fault tolerant design is added, on the basis of $\chi^{2}$ residual verification of the attitude determination system. Then, two variable step size state prediction methods are derived. Numerical simulation and analysis are described in section 4. Ultimately, in section 5 , the main conclusions and discussions are illustrated.

\section{Subsystem Kalman Filter}

\subsection{Gyros/GPS Kalman Filter}

The attitude determination residuals in quaternion form are set as main state variables, while the biases between the GPS's outputted attitude and estimated attitude are set as observation variables.

\subsubsection{State Equation}

The state equation is designed by kinematic attitude motion, and is described in quaternion form as:

$$
\dot{\mathbf{q}}=\frac{1}{2} \mathbf{q} \otimes \overline{\boldsymbol{\omega}}_{i b}^{b}
$$

where, $q$ is the quaternion form of attitude; and $\overline{\boldsymbol{\omega}}_{i b}^{b}=\left[\begin{array}{ll}0 & \left(\boldsymbol{\omega}_{i b}^{b}\right)^{T}\end{array}\right]^{T}$ means angular rate. The bias between real attitude quaternion $q$ and estimated attitude quaternion $\hat{\mathbf{q}}$ is set as $\Delta \mathbf{q}=\left[\begin{array}{llll}\Delta q_{0} & \Delta q_{1} & \Delta q_{2} & \Delta q_{3}\end{array}\right]^{T}$. Its incremental form is:

$$
\mathbf{q}=\hat{\mathbf{q}} \otimes \Delta \mathbf{q}
$$

Substituting equation (2) into equation (1), the following equation is obtained:

$$
\Delta \dot{\mathbf{q}}=\frac{1}{2} \Delta \mathbf{q} \otimes \hat{\boldsymbol{\omega}}_{i b}^{b}-\frac{1}{2} \hat{\boldsymbol{\omega}}_{i b}^{b} \otimes \Delta \mathbf{q}+\frac{1}{2} \Delta \mathbf{q} \otimes \Delta \boldsymbol{\omega}_{i b}^{b}
$$

where, $\Delta \boldsymbol{\omega}_{i b}^{b}$ denotes the residual error of the angular rate. According to the criterion of quaternion multiplication:

$$
\frac{1}{2} \Delta \mathbf{q} \otimes \hat{\boldsymbol{\omega}}_{i b}^{b}-\frac{1}{2} \hat{\boldsymbol{\omega}}_{i b}^{b} \otimes \Delta \mathbf{q}=\left[\begin{array}{l}
0 \\
\Delta \mathbf{q} \times \boldsymbol{\omega}_{i b}^{b}
\end{array}\right]
$$

The error quaternion is such a small value that $\Delta \mathbf{q}=\left[\begin{array}{llll}\Delta q_{0} & \Delta q_{1} & \Delta q_{2} & \Delta q_{3}\end{array}\right]^{T} \approx\left[\begin{array}{llll}1 & 0 & 0 & 0\end{array}\right]^{T}$, so

$$
\frac{1}{2} \Delta \mathbf{q} \otimes \Delta \hat{\boldsymbol{\omega}}_{i b}^{b}=\frac{1}{2} \Delta \boldsymbol{\omega}_{i b}^{b}+o\left(|\Delta \mathbf{q}|\left|\Delta \boldsymbol{\omega}_{i b}^{b}\right|\right)
$$

where, $o\left(|\Delta \mathbf{q}|\left|\Delta \boldsymbol{\omega}^{b}{ }_{i b}\right|\right)$ denotes a high-order infinitesimal value. Neglecting the second-order and subsequent infinitesimal segment, the linearized quaternion equation is described as:

$$
\begin{aligned}
& \Delta \dot{\mathbf{q}}_{13}=\left[\begin{array}{c}
\Delta \dot{q}_{1} \\
\Delta \dot{q}_{2} \\
\Delta \dot{q}_{3}
\end{array}\right]=\Delta \mathbf{q}_{13} \otimes \hat{\boldsymbol{\omega}}_{i b}^{b}+\frac{1}{2} \Delta \boldsymbol{\omega}_{i b}^{b} \\
& =-\left(\hat{\boldsymbol{\omega}}_{i b}^{b}\right)^{a} \Delta q_{13}+\frac{1}{2} \Delta \boldsymbol{\omega}_{i b}^{b} \\
& \Delta \dot{\mathbf{q}}_{0}=0
\end{aligned}
$$

The superscript $a$ represents the anti-symmetrical transformation process of a matrix.

$$
\left(\hat{\boldsymbol{\omega}}_{i b}^{b}\right)^{a}=\left[\begin{array}{ccc}
0 & -\hat{\omega}_{i b z}^{b} & \hat{\omega}_{i b y}^{b} \\
\hat{\omega}_{i b z}^{b} & 0 & -\hat{\omega}_{i b x}^{b} \\
-\hat{\omega}_{i b y}^{b} & \hat{\omega}_{i b x}^{b} & 0
\end{array}\right]
$$

On the basis of the gyro' observation equation, equation (6) is transformed into:

$$
\left\{\begin{array}{l}
\Delta \dot{\mathbf{q}}_{13}=-\left(\hat{\boldsymbol{\omega}}_{i b}^{b}\right)^{a} \Delta q_{13}-\frac{1}{2} d-\frac{1}{2} b-\frac{1}{2} \eta_{g} \\
\Delta \dot{\mathbf{q}}_{0}=0
\end{array}\right.
$$

$\eta_{g}$ represents the gyros' measurement noise. In this paper, the residual error of attitude is in quaternion form, and the gyros' time correlated drift d and gyros' constant drift $b$ are also selected as state variables:

$$
\mathbf{X}_{9 \times 1}=\left[\begin{array}{lll}
\Delta q_{13}^{T} & d^{T} & b^{T}
\end{array}\right]^{T}
$$

Thus, the state equation is described as below,

$$
\dot{\mathbf{X}}(t)=\mathbf{F}(t) \mathbf{X}(t)+\mathbf{W}(t)
$$

where, 
MAO Xinyuan Precise attitude determination strategy for spacecraft based on information fusion of attitude sensors: Gyros/...

$$
\begin{aligned}
& \mathbf{F}(t)=\left[\begin{array}{ccc}
-\left(\hat{\omega}_{i b}^{b}\right)^{a} & -0.5 I_{3 \times 3} & -0.5 I_{3 \times 3} \\
0_{3 \times 3} & -D_{\tau} & 0_{3 \times 3} \\
0_{3 \times 3} & 0_{3 \times 3} & 0_{3 \times 3}
\end{array}\right]_{9 \times 9} \\
& \mathbf{W}(t)=\left[\begin{array}{lll}
-0.5 \eta_{g} & \eta_{d} & \eta_{b}
\end{array}\right]^{T} \\
& \mathbf{D}_{\tau}=\operatorname{diag}\left[\begin{array}{lll}
\frac{1}{\tau_{x}} & \frac{1}{\tau_{y}} & \frac{1}{\tau_{z}}
\end{array}\right]
\end{aligned}
$$

$\tau$ is the correlated time constant. According to the standard Extended Kalman Filtering process:

$$
\mathbf{X}_{k}=\boldsymbol{\Phi}_{k, k-1} \mathbf{X}_{k, k-1}+\mathbf{W}_{k-1}
$$

where, $\boldsymbol{\Phi}_{k, k-1}$ is the state transition matrix from $t_{k-1}$ to $t_{k}$. Assuming that $t=t_{k-1}$,

$$
\boldsymbol{\Phi}_{k, k-1} \approx \mathbf{I}+\mathbf{F} T=\mathbf{I}+\left.\mathbf{F}\right|_{\mathbf{X}_{k-1}} T
$$

where, $T$ is the filtering cycle of filter; and $\mathrm{W}_{k-1}$ is the sequence of system noise, fulfilling the following condition:

$$
E\left\{\mathbf{W}_{k}\right\}=0, E\left\{\mathbf{W}_{k}, \mathbf{W}_{j}^{T}\right\}=\mathbf{Q}_{k} \delta_{k j}
$$

where, $\mathrm{Q}_{k}$ denotes the error variance matrix of state.

$$
\mathbf{Q}_{k}=\operatorname{diag}\left(0.25 \sigma_{g}^{2} \mathbf{I}_{3 \times 3} \quad \sigma_{d}^{2} \mathbf{I}_{3 \times 3} \quad \sigma_{b}^{2} \mathbf{I}_{3 \times 3}\right)
$$

$\sigma$ represents the mean square deviation of the gyros' drift white noise.

\subsubsection{Observation Equation}

The output of the GPS dual-baselines attitude determination system is in Euler-angle form. The residuals (difference between GPS observed attitude and estimated attitude) of GPS observation are selected as the observation variables in the Extended Kalman Filter.

$$
\mathbf{Z}=\left[\begin{array}{lll}
\gamma_{s}-\gamma_{e s t} & \theta_{s}-\theta_{e s t} & \psi_{s}-\psi_{e s t}
\end{array}\right]^{T}
$$

Then, the observation equation is described as below:

$$
\mathbf{Z}=\mathbf{h}(\mathbf{X}, t)+\mathbf{V}(t)
$$

Equation (17) is then linearized into:

$$
\mathbf{Z}_{k}=\mathbf{H}_{k} \mathbf{X}_{k}+\mathbf{V}_{k}
$$

where, $\mathrm{H}_{k}$ is the relevant matrix of the observed variables. Since the quaternion value twice the Euler angle when the attitude is of tiny value, the observation matrix is derived as:

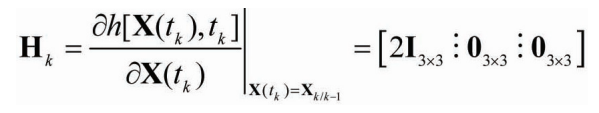

$\mathrm{V}_{k}$ is the sequence of observation noise, fulfilling the following condition:

$$
E\left\{\mathbf{V}_{k}\right\}=0, E\left\{\mathbf{V}_{k}, \mathbf{V}_{j}^{T}\right\}=\mathbf{R}_{k} \delta_{k j}
$$

$\mathrm{R}_{k}$ is the error variance matrix of the observation variables.

\subsection{Gyros/Star-sensor Kalman Filter}

In this filter, the residuals between the star-sensor's attitude observation (in quaternion form) and estimated attitude are set as observation variables. To correspond to the estimated attitude, it is necessary to transform the quaternion attitude into Euler angles, as described in the following equations:

Firstly, all the quaternion must be in unit vector form:

$$
\hat{\mathbf{q}}=\frac{q_{0}+q_{1} i+q_{2} j+q_{3} k}{\sqrt{q_{0}^{2}+q_{1}^{2}+q_{2}^{2}+q_{3}^{2}}}
$$

Thus, the Euler attitude can be calculated by:

$$
\left\{\begin{array}{l}
\phi=\arctan \left(\frac{2\left(q_{2} q_{3}+q_{0} q_{1}\right)}{q_{0}^{2}-q_{1}^{2}-q_{2}^{2}+q_{3}^{2}}\right) \\
\theta=\arcsin \left(2\left(q_{0} q_{2}-q_{1} q_{3}\right)\right) \\
\psi=\arctan \left(\frac{2\left(q_{1} q_{2}+q_{0} q_{3}\right)}{q_{0}^{2}+q_{1}^{2}-q_{2}^{2}-q_{3}^{2}}\right)
\end{array}\right.
$$

where, $\phi, \theta, \psi$ represent three Euler angles: yaw, pitch and roll, respectively.

\section{Kalman Filtering Strategy}

Two Kalman filtering strategies are utilized in this paper: the variable step size state prediction Kalman filter and the Federated Kalman Filter.

\subsection{Variable Step Size State Prediction Kalman Filter}

Different attitude sensors have different update rates, so it is crucial to utilize their data at maximum efficiency. In this paper, the gyros have a much higher update rate $(20 \mathrm{~Hz})$, than the GPS $(1 \mathrm{~Hz})$ and star-sensor $(5 \mathrm{~Hz})$. In the variable step size state prediction Kalman filtering process, if the observation data of the GPS and star-sensor are unavailable, only the filters' state variables are updated. As soon as the observation information is accessible, both state and observation variables are simultaneously updated.

\subsubsection{Prediction without Observation Update}

During the update gap of GPS and star-sensor at instant $t_{k-1}+i \Delta t(i=1,2, \ldots 19)$, these two sensors do not output any 
observation information. Then, the gyros' data is utilized, to predict state variables:

$$
\hat{\mathbf{X}}(i)=\boldsymbol{\Phi}(\hat{\omega}, \Delta t) \hat{\mathbf{X}}(i-1)
$$

where, $\Delta t$ is the filtering cycle, and is set as the gyros' update period (0.05s); and $\boldsymbol{\Phi}(\hat{\boldsymbol{\omega}}, \Delta t)$ is the state transition matrix. The prediction of error covariance matrix is described as:

$$
\mathbf{P}(i)=\boldsymbol{\Phi}(\Delta t) \mathbf{P}(i-1) \boldsymbol{\Phi}^{T}(\Delta t)+\mathbf{Q}
$$

\subsubsection{Observation Update}

Taking the star-sensor as an example, the star-sensor's output is possible at instant $t+4 \Delta t$, then the prediction process of state and observation variables is described as:

$$
\begin{aligned}
& \hat{\mathbf{X}}_{k / k-1}=\boldsymbol{\Phi}(\hat{\omega}, \Delta t) \hat{\mathbf{X}}(t+3 \Delta t) \\
& \mathbf{P}_{k / k-1}=\boldsymbol{\Phi}(\Delta t) \mathbf{P}(t+3 \Delta t) \boldsymbol{\Phi}^{T}(\Delta t)+\mathbf{Q} \\
& \mathbf{K}_{k}=\mathbf{P}_{k \mid k-1} \mathbf{H}_{k}^{T} \mathbf{H}_{k} \mathbf{P}_{k \mid k-1} \mathbf{H}_{k}^{T}+\mathbf{R}_{k}^{-1} \\
& \hat{\mathbf{X}}_{k}=\hat{\mathbf{X}}_{k / k-1}+\mathbf{K}_{k}\left(\mathbf{Z}_{k}-\mathbf{H}_{k} \hat{\mathbf{X}}_{k / k-1}\right)
\end{aligned}
$$

\subsubsection{Calibrating Quaternion and Gyros' Drift}

After acquiring the optimal prediction state:

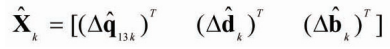

Calibrating the gyros' time related drift, and constant drift:

$$
\begin{aligned}
& \hat{\mathbf{d}}_{k}=\hat{\mathbf{d}}_{k, k-1}+\Delta \hat{\mathbf{d}}_{k} \\
& \hat{\mathbf{b}}_{k}=\hat{\mathbf{b}}_{k, k-1}+\Delta \hat{\mathbf{b}}_{k}
\end{aligned}
$$

Thus the calibrated state in quaternion form is:

$$
(\hat{\overline{\mathbf{q}}})_{k}=\left((\hat{\overline{\mathbf{q}}})_{k / k-1}\right) \otimes(\Delta \hat{\overline{\mathbf{q}}})_{k}
$$

Considering the constraint that the magnitude of quaternion equals to 1 :

$$
(\Delta \hat{\overline{\mathbf{q}}})_{k}=\left[\begin{array}{c}
\sqrt{1-\left(\Delta \hat{q}_{13 k}\right)^{T} \Delta \hat{q}_{13 k}} \\
\Delta \hat{q}_{13 k}
\end{array}\right]
$$

\subsection{Federated Kalman Filtering Strategy}

The Federated Kalman Filter consists of two independent subsystem filters, and one main filter, which fuses the subsystem filters' information, and outputs the global estimation. It introduces the feedback of global estimation, and leads to a higher fault tolerant capacity.

\subsubsection{Filtering Strategy}

Fig. 1 describes the FKF designed in this paper.

The output of the subsystem is the state estimation $X$ and error covariance matrix $P[9]$, and $\beta$ is the information allocation coefficient. In the main filter, all information is fused, based on the equations:

$$
\begin{aligned}
& \mathbf{P}_{f}^{-1}(k+1)=\sum_{i=1}^{2} \mathbf{P}_{i}^{-1}(k+1) \\
& \hat{\mathbf{X}}_{f}(k+1)=\mathbf{P}_{f}(k+1) \sum_{i=1}^{2} \mathbf{P}_{i}^{-1}(k+1) \hat{\mathbf{X}}_{i}(k+1)
\end{aligned}
$$

The significance of this method is obvious: if the precision of $\hat{\mathbf{X}}_{i}$ is very bad, namely $\mathrm{P}_{i}$ is very large, then its contribution $\left(\mathbf{P}_{i}^{-1} \hat{\mathbf{X}}_{i}\right)$ to final estimations should be very small. It is also significant to choose appropriate information allocation coefficients, while utilizing FKF [10]. In this paper, an adaptive coefficient choosing strategy is used:

$$
\begin{aligned}
& \beta_{1}=\frac{\sqrt{\operatorname{tr}\left(\mathbf{P}_{2}(k) \mathbf{P}_{2}(k)^{T}\right)}}{\sqrt{\operatorname{tr}\left(\mathbf{P}_{1}(k) \mathbf{P}_{1}(k)^{T}\right)}+\sqrt{\operatorname{tr}\left(\mathbf{P}_{2}(k) \mathbf{P}_{2}(k)^{T}\right)}} \\
& \beta_{2}=1-\beta_{1}
\end{aligned}
$$

where, $\operatorname{tr}$ represents the trace of a matrix.

Then, these feedbacks are utilized, to replace the subsystems' filtering information, when global estimation is obtained after fusion of the subsystem' state and error

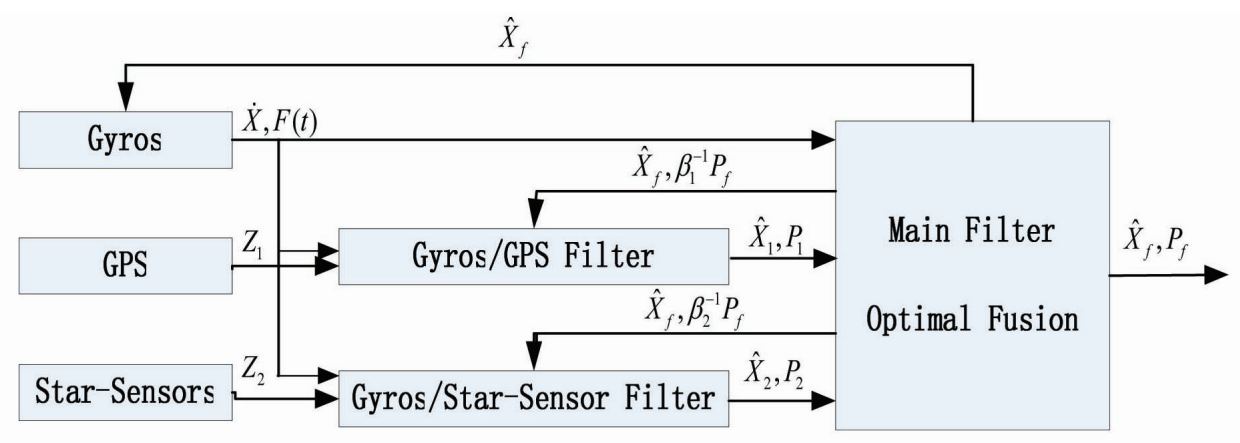

Fig. 1. Flow chart of the Federated Kalman filter 
covariance matrix in the main filter.

$$
\hat{\mathbf{X}}_{i}=\hat{\mathbf{X}}_{f}, \mathbf{P}_{i}=\beta_{i}^{-1} \mathbf{P}_{f}
$$

The update sequence of three sensors in 1 second is described in Fig. 2.

Obviously, all three sensors update at the instant when the GPS updates. It is a cycle of an integral FKF filtering process.

\subsubsection{Malfunction Warning Factor}

A malfunction warning factor, for checking whether or not a subsystem meets malfunction, is indispensable. There are two main checking methods: state $\chi^{2}$ verification strategy, and $\chi^{2}$ residual verification strategy. The former method does not introduce observation information, thus it is insensitive to detecting an observation sensor's malfunction, while the latter method overcomes this disadvantage [11]. The $\chi^{2}$ residual verification strategy is briefly described as below.

As for a subsystem Kalman filter, its residual is:

$$
\mathbf{r}_{k}=\mathbf{Z}_{k}-\mathbf{H}_{k} \hat{\mathbf{X}}_{k / k-1}
$$

where, the state vector is predicted by the equation:

$$
\hat{\mathbf{X}}_{k / k-1}=\boldsymbol{\Phi}_{k / k-1} \hat{\mathbf{X}}_{k-1}
$$

According to the filtering theorem, the residual is white noise with dispersion, as:

$$
\mathbf{A}_{k}=\mathbf{H}_{k} \mathbf{P}_{k / k-1} \mathbf{H}_{k}^{T}+\mathbf{R}_{k}
$$

Assuming a malfunction function as:

$$
\lambda_{k}=r_{k}^{T} \mathbf{A}_{k}^{-1} r_{k}
$$

It follows a $\chi^{2}$ distribution with $n$ (the dimensions of the observation vector) freedom. Then a malfunction warning factor is assumed as:

$$
l_{k}=\lambda_{k} / T_{D}
$$

where, $i$ denotes the index of subsystem filters. If $0<l_{k} \leq 1$, it means that no malfunction exists, while the opposite condition illustrates malfunction. $T_{D}$ represents the criterion of fault warning, and is determined by the statistical result of numerous calibrated experiments for a specified system [12].

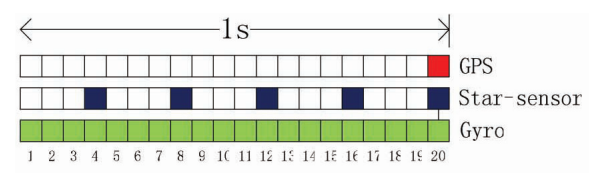

Fig. 2. The update sequence of three sensors in 1 second (color represents update instant)
Obviously, when malfunction occurs in a subsystem, its output should not be input into the main Kalman filter, or at least its weight should be decreased significantly. In this paper, the former conducting method is utilized, and the corrected output of FKF is utilized to replace the malfunctioned subsystem's observation information. Assuming that the first subsystem goes wrong, all its information should be ignored. Then the final estimations of the system state variables are:

$$
\hat{\mathbf{X}}_{g}=\hat{\mathbf{X}}_{2}
$$

Updating the malfunctioned subsystem's observation information as:

$$
\hat{\mathbf{Z}}_{1}=\mathbf{H}_{1} \hat{\mathbf{X}}_{g}
$$

These two procedures assist in decreasing the impact of malfunction to a maximum extent.

\section{Simulation and Analysis}

The main performance indexes of the three sensors are described in Table.1.

Fig. 3 describes the attitude determination accuracy, by utilizing the GPS only.

Obviously, the attitude determination accuracy is very low, before the double-differenced integer ambiguities are fixed by the LAMBDA method [13]. However, with the progressive improvement of the fixing success rate, the determination accuracy tends to be much better, with the best statistical accuracy of $\left(0.08^{\circ}, 0.08^{\circ}, 0.06^{\circ}\right.$, STD $)$ in Euler angles. Thus, the time of successfully resolving the integer ambiguity must be considered. In the following segment of this paper, all GPS observation data is collected, after the LAMBDA method has consecutively worked for more than 70 seconds (the time of $100 \%$ fixing success rate, got by 1000 times Monte Carlo

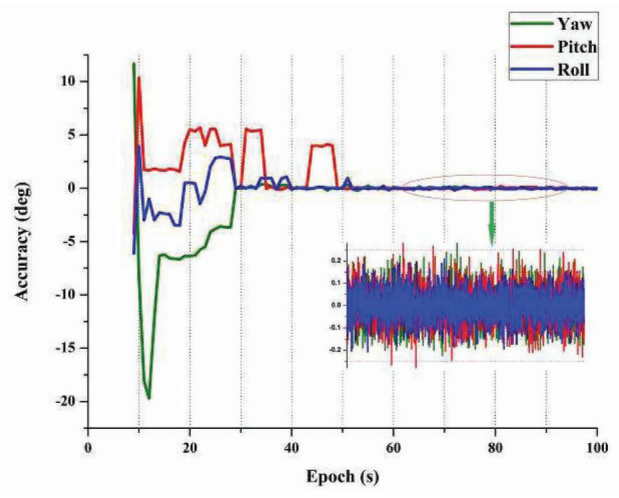

Fig. 3. Euler attitude determination accuracy by the GPS 
Table 1. Simulation parameters

\begin{tabular}{cccc}
\hline Sensor & Performance Index & Peak & Unit \\
\hline \multirow{3}{*}{ GPS Receiver } & PR observation accuracy & 0.2 & $\mathrm{~m}$ \\
& CP observation accuracy & 0.001 & $\mathrm{~m}$ \\
& System noise & 0.001 & $\mathrm{~m}$ \\
& Update rate & 1 & $\mathrm{~Hz}$ \\
& Baseline length & 1 & $\mathrm{~m}$ \\
\hline \multirow{3}{*}{ Gyros } & Constant drift & 3 & $\circ / \mathrm{h}$ \\
& Time correlative drift white noise mean square deviation & 0.1 & $\circ / \sqrt{h^{3}}$ \\
& Correlation time & 3600 & $\mathrm{~s}$ \\
& Time correlative drift initial value & 0.03 & $\circ / \sqrt{h^{3}}$ \\
& Measurement white noise mean square deviation & 0.02 & $\circ / \sqrt{h^{3}}$ \\
& Update rate & 20 & $\mathrm{~Hz}$ \\
\hline \multirow{2}{*}{ Star-sensor } & Observation accuracy & 3 & $\mathrm{c}$ \\
& Update rate & 5 & $\mathrm{~Hz}$ \\
\hline
\end{tabular}

statistical result).

Fig. 4 describes the attitude determination accuracy trends of the two subsystem filters and FKF.

In Fig. 4, the blue line stands for the accuracy of the Gyros/ GPS filter, the red line represents the Gyros/Star-sensor filter, and the green line denotes the final accuracy of the FKF. Clearly, the accuracy of the Gyros/Star-sensor filter converges quickly, while that of the Gyros/GPS converges much slower. FKF's accuracy almost coincides with that of the Gyros/Star-sensor. Their statistical results are illustrated in Table 2.

The final global estimation of FKF nearly approaches the Gyro/Star-sensor's result. That is mainly because both gyros and star-sensor are of much higher observation accuracy than the GPS, thus the Gyro/Star-sensor subsystem occupies a larger weight in FKF. In this process, the fault warning criteria $\left(T_{D}\right)$ of two subsystem filters are obtained.

Assume that from 1000s to 1200 s, the star-sensor is in

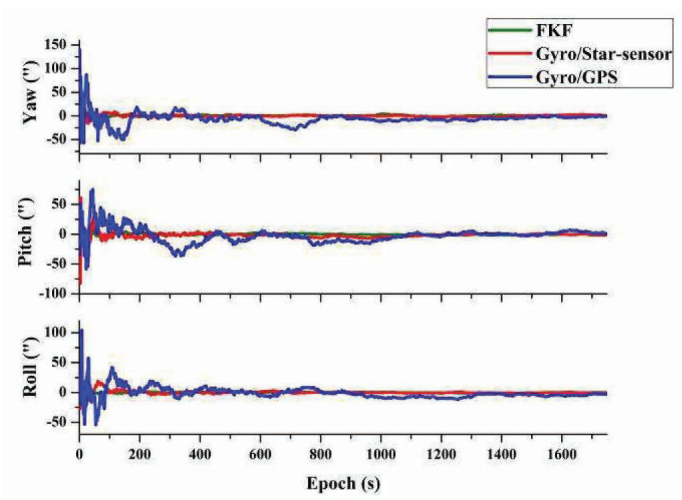

Fig. 4. The attitude determination accuracy trends by three Kalman filters fault, with malfunctioned observation accuracy at 10 times worse than usual. From 1400s to 1600s, the GPS runs out at the same level. The attitude determination accuracy of FKF and the two subsystem filters are described in Fig. 5.

Clearly, FKF is influenced drastically when the star-sensor malfunctions, because the FKF considers the star-sensor as normal accuracy, and does not adapt its weight. However, in this period, it still performs better than the malfunctioned Gyros/Star-sensor subsystem, indicating that the Gyros/GPS subsystem works effectively, to calibrate the FKF. When the GPS is in malfunction, the FKF is hardly being interrupted, because it occupies a slight weight in the FKF. As a whole, the FKF has a good malfunction tolerance performance. Table 3 describes the statistical results.

To check the performance of malfunction-modified procedure, another simulation with the same malfunction condition is analyzed. The varying trend of malfunction warning factors in this simulation circumstance is described in Fig. 6.

Obviously, the malfunction warning factors alarm all malfunction periods precisely and correctly. Adding malfunction tolerant design to the FKF, the attitude determination accuracy compared with no-malfunction FKF, and malfunction un-modified FKF, is described in Fig. 7.

Table 2. Statistical result of Euler attitude (1800s, STD)

\begin{tabular}{llll}
\hline Strategy/Attitude & Yaw (") & Pitch (") & Roll (") \\
\hline Gyro/Star-sensor & 1.856 & 2.254 & 1.565 \\
Gyro/GPS & 12.440 & 12.317 & 9.442 \\
FKF & 1.563 & 2.194 & 1.513 \\
\hline
\end{tabular}


It can be seen from Fig. 7 that the FKF with the modified malfunction procedure achieves better accuracy than the un-modified one. The statistical results are described in Table 4.

Clearly, after being modified by the strategy proposed in this paper, the FKF's final attitude determination accuracy approximately approaches the no-malfunction situation. This indicates that the strategy effectively avoids the impact of malfunction.

\section{Conclusion and Discussion}

In this paper, an attitude determination strategy for spacecraft based on the information fusion of Gyros/ GPS/Star-sensor was demonstrated. In the information fusion process, two subsystem Kalman filters were firstly established. To cope with the problem of the different sensors' update rates, they were both designed with variable step size state prediction strategies. Finally, an FKF with malfunction warning design was established; the output of the subsystem filters' state and error covariance matrix were selected as the input of the main filter. The final result indicated that the FKF effectively negated the impact of possible malfunction.

This paper analyzed these problems based on the numerical simulation process; however, it did not take into consideration a sophisticated model of each sensor, and the simulation circumstance was of relatively ideal quality.

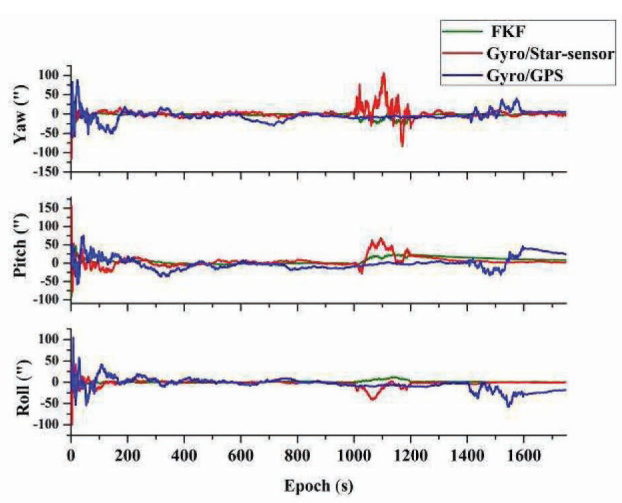

Fig. 5. The attitude determination accuracy, when malfunctions occur in a subsystem

Table 3. Statistical result of the Euler attitude (1800s, STD)

\begin{tabular}{llll}
\hline Strategy/Attitude & Yaw (") & Pitch (") & Roll (") \\
\hline Gyro/Star-sensor & 14.061 & 16.591 & 8.592 \\
Gyro/GPS & 14.314 & 17.163 & 13.927 \\
FKF & 5.175 & 8.849 & 3.030 \\
\hline
\end{tabular}

In real applications, the pre-processing of real space-borne sensor data is another important problem. Besides, the FKF designed in this paper could not consistently obtain optimal estimation. These issues should be paid attention to in future research.

\section{Acknowledgement}

This work is supported by the Natural Science Foundation of China (NSFC), under Grant No.61173077, and the National High-Tech. R\&D Program of China (863), under Grant No.2011AA120505.

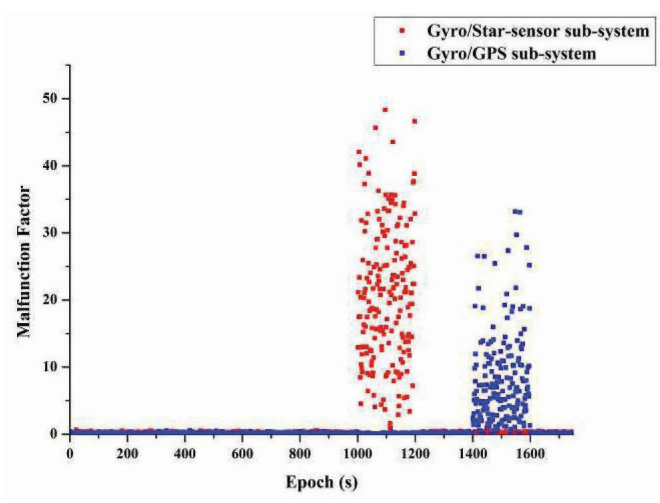

Fig. 6. Malfunction warning factors of two subsystem filters

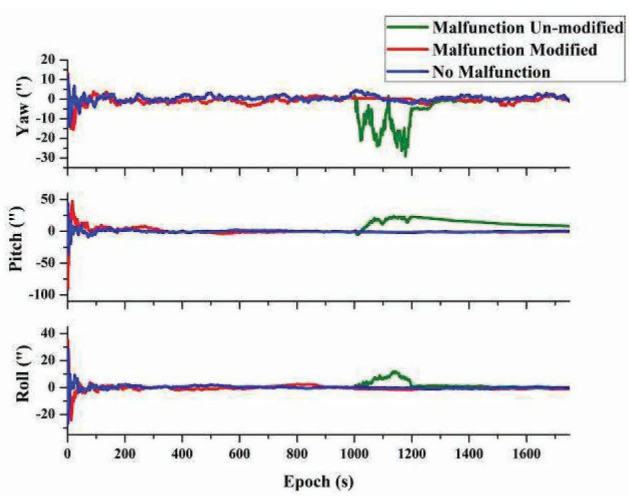

Fig. 7. Three kinds of FKF accuracy

Table 4. Statistical result of the Euler attitude (1800s, STD)

\begin{tabular}{llll}
\hline Strategy/Attitude & Yaw (") & Pitch (") & Roll (") \\
\hline Malfunction Un-modified & 5.176 & 8.849 & 3.030 \\
Malfunction Modified & 1.795 & 5.012 & 2.157 \\
No malfunction & 1.563 & 2.194 & 1.513 \\
\hline
\end{tabular}




\section{References}

[1] Itzhack, Y., Bar-Itzhack, and Richard, R., Harman, "Optimized TRIAD Algorithm for Attitude Determination", Journal of Guidance, Control, and Dynamics, Vol. 20, No. 1, 1997, pp. 208-211.

DOI: $10.2514 / 2.4025$

[2] Itzhack, Y., Bar-Itzhack, "New Method for Extracting the Quaternion from a Rotation Matrix", Journal of Guidance, Control, and Dynamics, Vol. 23, No. 6, 2000, pp. 1085-1087.

DOI: $10.2514 / 2.4654$

[3] Jonghee, Bae, and Youdam, Kim, "Attitude Estimation for Satellite Fault Tolerant System Using Federated Unscented Kalman Filter", International Journal of Aeronautical and Space Sciences, Vol. 11, No. 2, 2010, pp. 80-86.

DOI: 10.5139/IJASS.2010.11.2.080

[4] T.M.A., Habib, "A Comparative Study of Spacecraft Attitude Determination and Estimation Algorithms (A Costbenefit Approach)", Aerospace Science and Technology, 2012.

URL : http://dx.doi.org/ 10.1016/j.ast.2012.04.005

[5] Demoz, Gebre-Egziabher, Roger, C., Hayward, and J., David, Powell, "Design of Multi-sensor Attitude Determination Systems", IEEE Transactions on Aerospace and Electronic Systems, Vol. 40, No. 2, 2004, pp. 627-649.

[6] R.E., Kalman, "A New Approach to Linear Filtering and Prediction Problems", Journal of Basic Engineering (ASME), Vol. 82D, 1960, pp. 35-45.
[7] J.D., Pearson, and S., Reich, "Decomposition of Large Optimal-control Problems", Proceedings of the Institution of Electrical Engineers, Vol. 114, No. 6, 1967, pp. 845-851.

[8] Guo Kang, Du Xiaojing, and Mao Xinyuan, "Research on Multi Update Rate Method of Precise Satellite Attitude Determination Based on Gyro and Star-Sensor", AIAA Guidance, Navigation, and Control Conference, Portland, USA, 2011.

[9] Jianguo Yan, Dongli Yuan, Xinmin Wang, et al, "A Study of Information Fusion Based on Federated Kalman Filtering", Proceedings of the 6th World Congress on Intelligent Control and Automation, Dalian, China, 2006.

[10] Tao Junyong, Qiu Jing, Wen Xisen, et al, "Adaptive Federated Filter Model and Its Application in SINS/GPS Integrated Navigation System for Vehicle", Information and Control, Vol. 29, No. 2, 2000, pp. 168-172.

[11] Wu Xunzhong, Zhou Jun, and Qiu Kai, "Study of A Robust Federated Filtering Algorithm Based on Fault Factor Function", Journal of Astronautics, Vol. 27, No. 1, 2006, pp. 57-60.

[12] Liu Zhun, and Chen Zhe, "New Fault Detection Structure and Algorithm Based on Federated Filter", Journal of Beijing University of Aeronautics and Astronautics, Vol. 28, No. 5, 2002, pp. 550-554.

[13] P.J.G., Teunissen, “The Least-squares Ambiguity Decorrelation Adjustment- A Method for Fast GPS Integer Ambiguity Estimation", Journal of Geodesy, Vol. 70, No. 1-2, 1995, pp. $65-82$.

DOI: $10.1007 /$ BF00863419 